\title{
Laser Surface Modification to Enhance Brazing Joints.
}

\author{
Nerea OTERO*1 ${ }^{*}$ Pablo ROMERO ${ }^{* 1}$, José Carlos SOTELO ${ }^{* 1}$ and Alejandro GONZÁLEZ ${ }^{* 1}$ \\ ${ }^{* 1}$ AIMEN Technology Center, Laser Applications Centre (CAL); c/ Relva 27A-Torneiros; 36410 \\ Porriño (Pontevedra), Spain \\ E-mail: notero@aimen.es
}

Laser generated topographical and chemical modifications are introduced on metallic surfaces to affect the surface tension forces driving the brazing and soldering processes on different alloys. The introduced regular surface condition consists of bimodal roughness arranged according to periodic patterns (multiscale texturing), by exploiting combination of melting, ablation and reaction with atmosphere. The use of multistep scanning patterns to form highly anisotropic textures, and their capability to influence the flow of molten metal during the wetting phase of braze joining and soldering is investigated in this work.

DOI:10.2961/jlmn.2013.02.0004

Keywords: Laser surface texturing, brazing, molten metal wettability.

\section{Introduction}

Braze joining (or brazing) is the technique of choice in many highly demanding industries to produce fatigue resistant, durable and dependable joints between two metals, or between metals and ceramics [1]. It is a mature and well established technology in aerospace, automotive or energy industry. The process is driven by capillary forces which appear among the two surfaces to be joined and the filler material in molten state. The flow properties of the filler material are strongly influenced by the surface roughness, and surface condition is a usual source of process defects and deviations [2].

This work presents a simple procedure for laser assisted generation of a controlled and regular surface condition consisting in bimodal roughness arranged according to periodic patterns, by using nanosecond pulse patterns in mixed ablation-melting regime to induce high aspect ratio and dual roughness features [3]. The surface can be defined in terms of autocorrelation length for each of the roughness components and spatial distribution of the dual texture pattern.

The dual roughness provides the possibility to simultaneously improve various brazing process characteristics, like surface wettability reducing wetting defects, distance between parts to be joined, and improve the mechanical resistance of the joint.

\section{Motivation}

Brazing and soldering are joining technologies where a filler material is heated above its liquidus temperature, which is lower than the melting point of the parts to be joined, forming a physical and metallurgical bond between both parts by solidification and diffusion processes. The filler material movement in the joining area is governed by two driving forces: capillarity and surface tension.
Many quality issues in brazing come from the molten filler metal spread mechanism, particularly in joints with complex geometry:

- With high surface tension, brazing alloys finds difficulties to spread in large areas or climb not horizontal surfaces.

- If filler material flows off from planned joining areas, it forms drops and accumulations which cause damages in components.

- If filler material is not filling the whole joining area, joint strength will be lower than expected and a crack initiator may appear under cyclic loading.

- Excess of material, lack of material or bad clearance result in dangerous defects and poor fatigue performance.

These problems have to be considered as part of joint designing. Some commercial solutions proposed imply to generate a wall-like-layer with a limit off material added in form of paste or additional part or an oxidised grove. All these solutions have proven very effective to limit the material spread and reduce certain defects, but they are highly intrusive and require an extra operation which, in most cases, is hard to automate.

\subsection{Fundamentals of wettability}

Young [4] proposed a simple way of describing a liquid-solid interface within a gas atmosphere, to determine the shape of the formed drop:

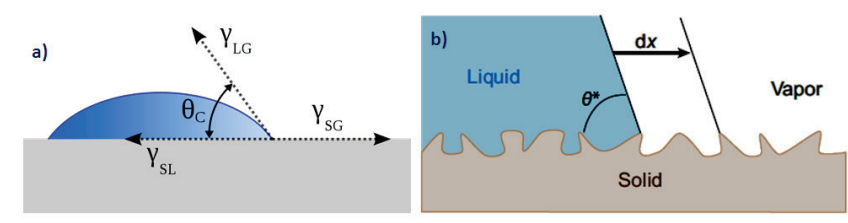

Figure 1: Wetting angle according to: a) Young, b) Wenzel 
In this simple model, drop shape depends only on material properties (Figure 1.a), resulting in surface tension values, according to [Eq.1], which can be read as a simple static equilibrium equation, with $\gamma_{A B}$ the A-B interfacial force and $\theta$ the wetting angle:

$$
\gamma_{S G}=\gamma_{S L}+\gamma_{L G} \cos \theta
$$

For most brazing conditions, the value of the wetting angle $\theta$ lies in the $18-23^{\circ}$ range and should be the same for the same filler, base and atmosphere composition. Anyway, it is verified in the practice that surface condition affects the actual wetting angle (Figure 1.b), so a more precise model should be used.

$$
\cos \theta^{*}=r \cos \theta=r \cdot \frac{\gamma_{L G}-\gamma_{S L}}{\gamma_{S G}}, \mathrm{r}=\mathrm{S}_{\mathrm{A}} / \mathrm{S}_{\mathrm{P}}
$$

The Wenzel model uses the roughness factor $(0<\mathbf{r} \leq 1)$, defined as the ratio between the total and projected surface (specific surface, [Eq.2]). This model predicts that roughness amplifies the natural behaviour of the interfaces, that is, hydrophilic surfaces will be more hydrophilic with higher roughness [5].

This model works well for moderate roughness. However, in surfaces with very high $\mathbf{r}_{\mathbf{t}}$ values, air pockets forms below the drop. The energy balance favours sticking to the solid instead of creating liquid/vapor interfaces to jump over the air pocket (Figure 2), so an apparent hydrophilic behaviour is induced. The Cassie-Baxter model predicts the equilibrium wetting angle as shown in [Eq.3], where $\boldsymbol{\Phi}_{\mathbf{S}}$ is the surface fraction in contact with the liquid.

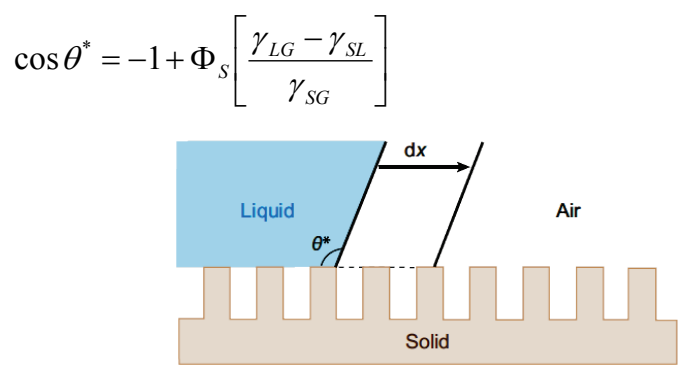

Figure 2: Cassie state

As a first approach, surface roughness under the Cassie switching condition will help filler metal spreading and ensure filling. On the other hand, high $\mathbf{r}_{\mathbf{a}}$ roughness will provide a purely mechanic stop-off effect. This general concept is, anyway, insufficient for defining the surface required for a full control of the molten metal spread as required for technological applications of custom surface texturing to the improvement of brazing and soldering quality. For this reason, a better definition is sought in the next epigraph.

\subsection{Dynamic wettability in molten metal}

Brazing is driven by a combination of wetting and capillary flow (Figure 3), as well as diffusion (partial dealloying and dilution). In the following, only wetting and capillarity will be considered, and a simplification will be applied to avoid considering diffusion, as it involves time dependent chemistry.

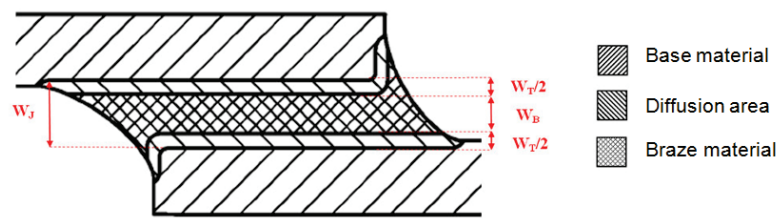

Figure 3: Wetting behaviour of filler metal in a braze joint

The dynamic process from the applied filler material to the final equilibrium stage involves several stages. A gravity driven stage, right over the solidus temperature, when Bond number $\mathrm{B}_{0}[$ Eq.4] is well over 0.02 , so molten metal mass governs over capillary and surface tension forces. A second stage, inertial, still in incomplete phase change, with spreading driven by the increasing volume of available liquid. A third stage of viscous flow, driven by capillary forces. And a final asymptotic stage, characterized by source metal depletion, where cohesion and surface tension forces dominate.

$$
B_{0}=g \rho(V / A)^{2} / \sigma
$$

(where $g$ is the gravitational acceleration, $\rho$ the fluid density, $V / A$ is the equivalent characteristic length scale or capillary radius, calculated as volume $V$ of wetting liquid over its projection area $A$, and $\sigma$ is the surface tension).

The final shape of the interface will mainly depend on the final step, while the area of the affected zone will depend on third and forth stages. Both characteristics are crucial to braze joint quality, so Stage 3 and 4 will be analyzed to determine the optimal surface characteristics.

The main characteristic sought is the flow anisotropy, to force a flow of molten metal in preferential directions, avoiding undesired overflows in the perpendicular direction, and granting the full surface wetting. Hence, surface texture will be highly anisotropic. For defining molten metal spread on anisotropic rough surface, the tortuosity factor is useful: $\mathbf{x}^{*}=\mathbf{x} \cdot \tau_{\mathbf{x}}$, with $\mathbf{x}$ the projected dimension (valid for Young model angle calculation) and $\mathbf{x}^{*}$ the real spread travel of the liquid on the surface, affected by directional roughness in the $\mathbf{x}$ axis. With $\tau_{\mathbf{y}}>>\tau_{\mathbf{x}}$, the texture can be defined as channels in the $\mathbf{x}$ direction. 


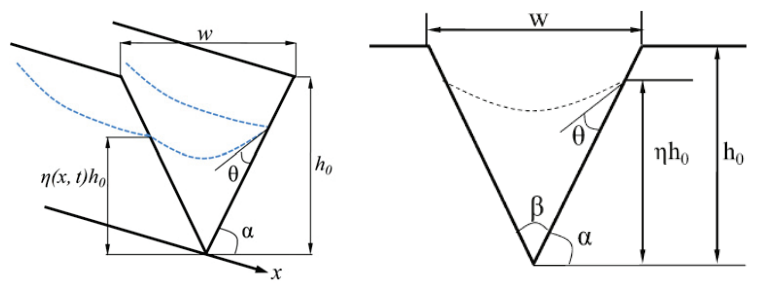

Figure 4: Geometric characterization of generic microchannels

Considering a surface generically constituted as an array of microchannels (Figure 4), geometrically characterized as shown in Figure 5, then the volumetric flow $\mathbf{q}\left(\mathbf{x}^{*}, \mathbf{t}\right)$ can be written as expressed in Eq.6, where $-\partial \mathbf{p} / \partial \mathbf{x}^{*}$ is the capillary pressure gradient (spread driving force), $\boldsymbol{\mu}$ is the dynamic viscosity of the molten metal, and $\boldsymbol{s}\left(\mathbf{x}^{*}, \mathbf{t}\right)$ is the cross section area of molten metal at point $\mathbf{x}^{*}$, which can be expressed in terms of texture geometry and natural wetting angle $\boldsymbol{\theta}$ according to Eq.7.

$$
\begin{aligned}
& q\left(x^{*}, t\right)=\frac{\aleph^{2}\left(x^{*}, t\right)}{8 \pi \mu} \cdot\left(-\frac{\partial p}{\partial x^{*}}\right) \\
& \aleph^{2}\left(x^{*}, t\right)=\left[\eta\left(x^{*}, t\right) h_{o}\right]^{2} \Phi(\alpha, \theta)
\end{aligned}
$$

Capillary pressure, on the other hand, is expressed by [Eq.8] as a function of natural wetting angle $\theta$, the topographic characteristics and the topography filling behaviour of the metal, expressed here by the relative metal height $\eta$. The value of can be that of Young, Wenzel or Cassie model, according to the surface condition.

$$
p_{\text {capillar }}=\frac{\sigma_{L V} \cdot \sin (\alpha-\theta) \cdot \tan \alpha}{\eta(\alpha-\theta) \cdot h_{0}}
$$

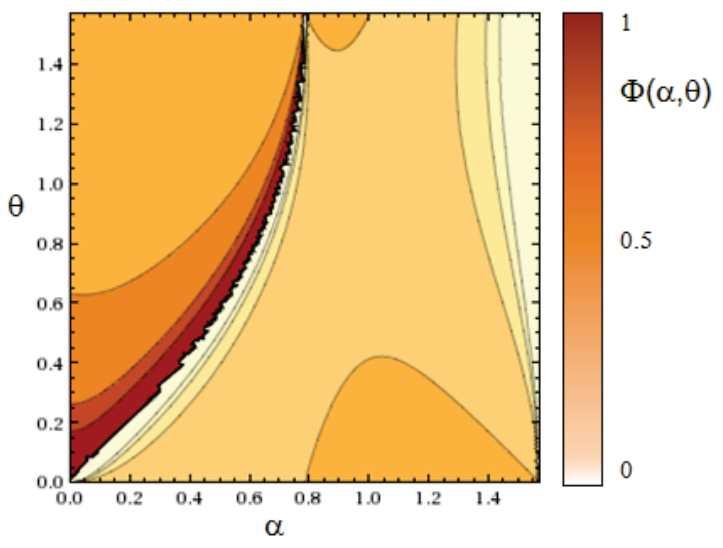

Figure 5: Graphical depiction of the $\Phi(\alpha, \theta)$ function

Finally, the spread limit or metal reach in one direction can be expressed as in [Eq.9], allowing to determine the desired surface geometry.

$$
x=x_{0} \frac{\eta_{0, x} h_{0}}{\tau_{x}^{2} \pi} \frac{\sigma_{L V}}{\mu} \Phi(\alpha, \theta)
$$

\subsection{Desired surface features and way to obtain them}

From the previous section, it can be derived that the surface features to be developed are clearly differentiated if the area is to be textured for conducting the metal spread, or for retaining the flow (stop-off like behaviour).

For conducting the flow, a very high increase in tortuosity is required in the perpendicular direction, that is, very high contrast in the directional $\mathbf{r}_{\mathbf{q}}$ and $\mathbf{r}_{\mathbf{k}}$ roughness parameters. For increasing the $\cos \theta^{*}$ value, a secondary roughness within the primary structure will improve the cross section area of channel filling and the capillary pressure.

For most filler metal and base metal combinations, this could be fulfilled by a channel height in the range of 50 to 100 microns, and secondary roughness under the Cassie limit, which could lie in the 5-10 microns.

For flow stopping the strategy can be based in flow pinning structures (high secondary roughness of $\mathbf{r}_{\mathbf{a}}$ under the Cassie switching limit) with metal-phillic behaviour (hydrophilic property of molten metal) surrounded by flat surfaces, to induce high hysteresis. The metal flow pinning structures should be well over 100 microns in height and tens to hundreds of microns in spacing to be effective in the range of surface forces which are being considered in brazing. Another effective melt-stop strategy is the contact angle contrast effect, with highly wettable surface right in contact with a Cassie-Baxter regime inducing surface. Then the flow should stop right in the limit between both areas, according to the shown theoretical frame. The following experimental work will verify these assumptions.

\section{Laser texturing process}

Two different laser sources were used to attain surface structures with different shapes and size ranges.

A Q-Switched $\mathrm{Nd}: \mathrm{YVO}_{4}$ green laser $(\lambda=532 \mathrm{~nm})$, $\mathrm{M} 2<1.2$, pulse duration $<10 \mathrm{~ns}$, up to $200 \mathrm{kHz}$ rep-rate, provided up to $400 \mu \mathrm{J}$ energy per pulse. The beam is delivered through a galvoscanner head with $254 \mathrm{~mm}$ focal length field optics, providing a spot size FWHM of $60 \mu \mathrm{m}$ in diameter and a resulting instantaneous power density up to $14 \mathrm{MW} / \mathrm{mm}^{2}$.

A single mode fiber laser (wavelength $\lambda=1070 \mathrm{~nm}$ ) has been used, with a fiber core diameter of 11 microns and a maximum $\mathrm{CW}$ power of $400 \mathrm{~W}$. Diode modulation up to 50 $\mathrm{kHz}$ is available for this laser. Beam is guided through a galvoscanner, the spot size is under 40 microns in diameter, and maximum scanning speed is $1000 \mathrm{~mm} / \mathrm{s}$.

These laser sources were used for texturing the flat surface of an aeronautic grade Nickel alloy (Nimonic N75) 
with features with different shapes and sizes in the micron range. To obtain the required sub-beam size features, partially overlapping scanning strategies were repeated with sub-beam size displacements to get the desired accumulation of molten metal displaced by the recoil pressure.

Contact angle and joint strength with and without the textured surfaces were measured using a Ni602 brazing alloy. Compositions of both base and filler metal are listed in the Table 1.

Table 1: Brazing material composition
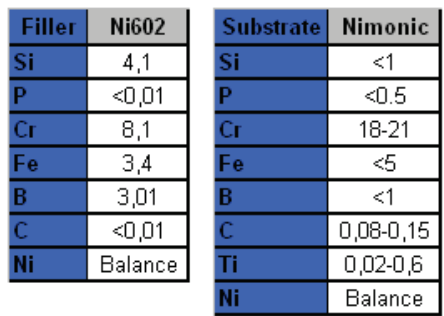

\section{Experimental procedure}

Two different experiments were performed, to check the effect of laser texturing on brazing and soldering processes.

For brazing experiment, six different kinds of patterns were generated by laser surface texturing. To perform the different philosophies and effects based in laser-material interaction and relative laser beam geometrical movement were applied to generate the following texture designs:

- N1: fine hexagonal 60x60 $\mu \mathrm{m}$ mesh generated by nanosecond pulses. Ablation creates microcavities in the metal, while the recoil pressure and temperature causes movement of molten material creating a wall between the cavities, increasing the total apparent aspect ratio and secondary roughness.

- N2: fine riblets pattern with 200 microns period generated by nanoseconds pulses. In this case the movement of the molten material was enhanced in one direction and suppressed in the perpendicular direction. Laser scans with high pulse overlapping generating a grove and a displacement of molten material to one side. In each cycle, scanning trajectory is displaced 3 microns perpendicularly, increasing the molten material wave and improving secondary directional roughness.

- K1: 400x400 $\mu \mathrm{m}$ dimple mesh generated by the relative movement of a CW irradiation over the surface. By a radial scanning movement of 300 microns each $45^{\circ}$, height- base relation is increased. Effect is enhanced by repeating 10 times the scanning pattern.

- K2: $800 x 800 \mu \mathrm{m}$ dimple mesh generated by the relative movement of a continuous irradiation over the surface. Similar to K1, in this case effect is enhanced lowering the repetition rate but increasing speed and decreasing angle increment to $9^{\circ}$.

- M1: riblets pattern with 400 microns period generated by the movement of a continuous irradiation over the surface, modulating the energy along the scanned line with a 400 microns period to produce the movement and accumulation of the molten material in the less affected areas. Ribletted pattern is generated.
- P1: Hexagonal pattern of dimples, 300 microns pitch, generated by the ejection of melted material caused by high brilliance millisecond pulses with high energy density. Plasma expansion in each pulse causes localized force against the surrounding atmosphere and, after the plume is dissipated, a suction force that attracts the molten material underneath, and hence an elevation. The height of the elevation is controlled by the number of repetitions.

These textures were generated in a $30 \times 30 \mathrm{~mm}$ area of the Nimonic N75 surface. Power-scanning parameters were adapted to the thermophysical properties of the base material, to attain a mixed melting-ablation or meltingvaporization processing regime.

Table 2: Power-scanning parameters

\begin{tabular}{|c|c|c|c|c|c|c|c|}
\hline \multicolumn{8}{|c|}{ Q-Switched Vanadate Laser } \\
\hline Id. & Texture & Dim. $(\mu \mathrm{m})$ & $E(\mu)$ & $V(\mathrm{~mm} / \mathrm{s})$ & Pulse (ns) & Rep rate & Cycles \\
\hline N1 & $\begin{array}{c}\text { Fine hexag. } \\
\text { mesh }\end{array}$ & $60 \times 60$ & 350 & 1200 & 10 & $20 \mathrm{kHz}$ & 70 \\
\hline N2 & Fine riblets & 200 & 150 & 650 & 10 & $60 \mathrm{kHz}$ & 150 \\
\hline \multicolumn{8}{|c|}{ CW Fibre laser } \\
\hline Id. & Texture & Dim. $(\mu \mathrm{m})$ & $P(w)$ & $V(\mathrm{~mm} / \mathrm{s})$ & Pulse (ms) & Rep rate & Cycles \\
\hline M1 & Riblets & 400 & 80 & 800 & 12 & $1 \mathrm{~Hz}$ & \\
\hline K1 & $\begin{array}{c}\text { Coarse } \\
\text { square mesh }\end{array}$ & $400 \times 400$ & 40 & 500 & $\mathrm{CW}$ & - & 10 \\
\hline K2 & $\begin{array}{c}\text { Coarse } \\
\text { square mesh }\end{array}$ & $800 \times 800$ & 40 & 800 & $\mathrm{CW}$ & - & 4 \\
\hline P1 & Dimples & $300 \times 260$ & 150 & & 0,5 & $60 \mathrm{~Hz}$ & 7 \\
\hline
\end{tabular}

Resulting textures are shown in figure 6:

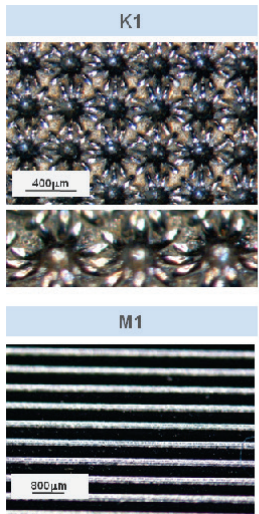

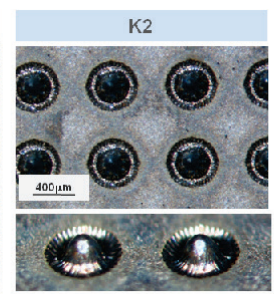

N1

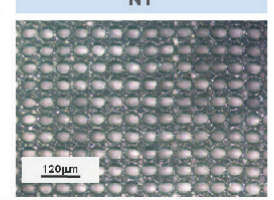

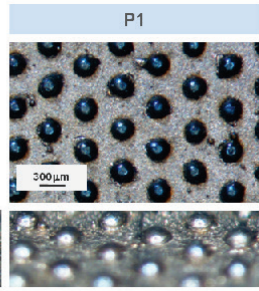

N2

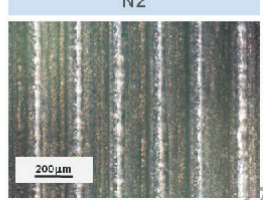

Figure 6: Laser induced textures on Ni alloy surface

The material for soldering test was a typical soldering filler material based on a Sn alloy that would melt over a brass textured surface. Base material was a BS2874 CZ121 with $58 \% \mathrm{Cu}, 3 \% \mathrm{~Pb}$ y $39 \% \mathrm{Zn}$. Before the treatment it was previously polished. Filler material had a $63 \% \mathrm{Sn}$ and a eutectic point of $183.3^{\circ} \mathrm{C}$.

In some cases it was needed an extra commercial flux for generic soldering applications. 2 main patterns were generated on the Brass surface: a cage like confining area surrounded by a N1 texture and a N2 riblets patterned area. Dimensions and results are listed in the Table 3. 
Table 3: Laser induced surfaces for soldering experiments

\begin{tabular}{|c|c|c|c|}
\hline Series & Characteristics & Sample \# & Structure \\
\hline \multirow{3}{*}{ A } & \multirow{3}{*}{$\begin{array}{l}10 \times 10 \mathrm{~mm} \mathrm{Sq} . \\
145 \mathrm{mg} \mathrm{SnPb}\end{array}$} & $1 \& 2$ & Meshed \\
\hline & & $3 \& 4$ & Rlbletted \\
\hline & & $5 \& 6$ & Bumlshed \\
\hline \multirow{3}{*}{ B } & \multirow{3}{*}{$\begin{array}{c}10 \times 10 \mathrm{~mm} \mathrm{Sq} . \\
30 \mathrm{mg} \mathrm{SnPb}\end{array}$} & $1 \& 2$ & Meshed \\
\hline & & $3 \& 4$ & Ribletted \\
\hline & & $5 \& 6$ & Burnished \\
\hline \multirow{6}{*}{$\mathrm{v}$} & \multirow{3}{*}{$\begin{array}{l}\text { Laser written boundary (laser } \\
\text { Induced stop-off) }\end{array}$} & $1 \& 2$ & Meshed square 10 $\mathrm{mm}$ (crossing IInes) \\
\hline & & $3 \& 4$ & Ribleted square $10 \mathrm{~mm}$ (crossing IInes) \\
\hline & & $5 \& 6$ & Meshed Boundary (sq. $6 \times 6 \mathrm{~mm}$ ) \\
\hline & \multirow{3}{*}{$145 \mathrm{mg}$ SnPb } & $7 \& 8$ & Rlbletted Boundary (sq. $6 \times 6 \mathrm{~mm}$ ) \\
\hline & & $9 \& 10$ & Meshed boundary $(4 \times 4 \mathrm{~mm})$ \\
\hline & & $11 \& 12$ & Rlbletted boundary $(4 \times 4 \mathrm{~mm})$ \\
\hline \multirow{3}{*}{$\mathrm{R}$} & \multirow{3}{*}{$\begin{array}{l}\text { Square } 10 \times 10 \mathrm{~mm} \text { Argon } \\
\text { atmosphere (145mg SnPb) }\end{array}$} & $1 \& 2$ & Mesh \\
\hline & & $3 \& 4$ & Riblets \\
\hline & & $5 \& 6$ & M. Base \\
\hline
\end{tabular}

The following images (Figure 7) display some examples of the laser induced surfaces prepared on brass for soldering experiments.
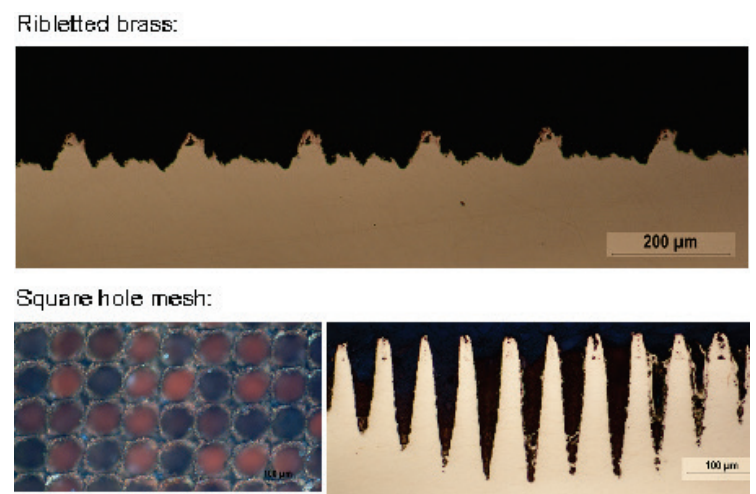

Figure 7: Soldering textures

\section{Results and discussion}

\subsection{Brazing results and discussion}

For the brazing experiments on textured and nontextured Nimonic samples, a quantity of Ni-based filler metal, in the form of Ni 602 paste, was deposited on each patterned surface. Samples were then brought to high temperature in a vacuum oven. In the following plot (Figure 8), the controlled pressure and temperature cycle is shown, which was applied to all samples under study.

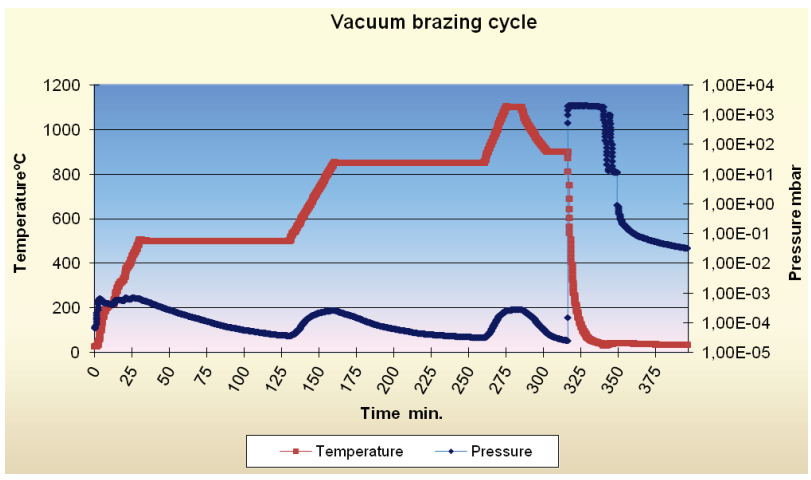

Figure 8: Vacuum brazing cycle

For a dimensional study of the tested surfaces a profilometry analysis was performed. Due to the complex geometry in both directions of the structure, a surface mapping was performed using a Wyko-NT1100 optical profilometer using an objective with a magnification of $5 \mathrm{x}$ and a FOV of 1x (in the case of $\mathrm{N} 1$ a magnification of 20x was

needed). Topographic view allowed a better understanding of the surface shape. The images of the topographic results are shown in the following figures (Figure 9 and Figure 10).

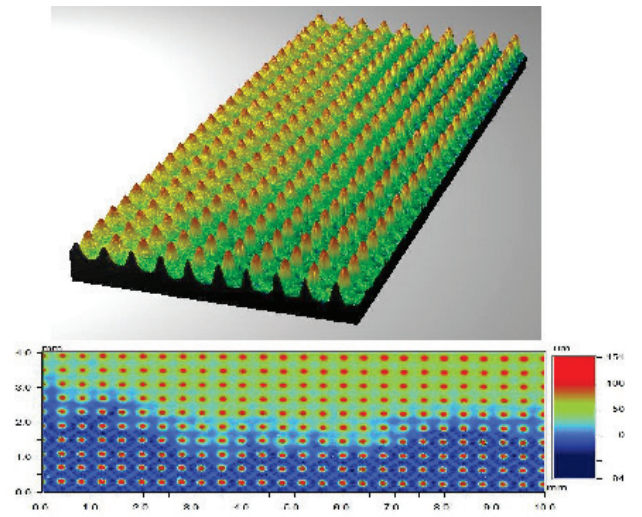

Figure 9: K1 topographic test

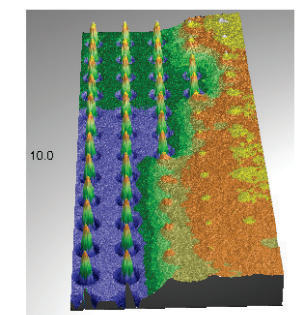

$4.0 \mathrm{~mm}$

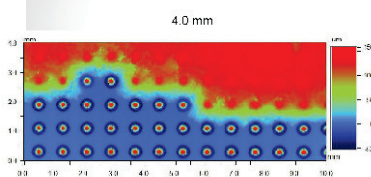

Figure 10: a) K2 topographic test, b) M1 topographic test

K2 sample induces a high hysteresis Cassie effect (Figure 11). In a Cassie state, a drop is likely to pin on the edges of the defects as we displace it. The drop distortion, and the energy stored in this deformation fixes the amplitude of the hysteresis [5].

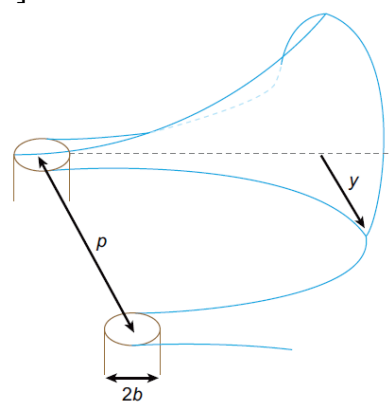

Figure 11: Pinning effect

\subsection{Soldering results and discussion}

Spread of solder on textured and non textured brass surfaces were performed, with two distinct objectives: To visualize the spreading mechanism of the molten filler material on the surface and to demonstrate the possibilities of the patterned surfaces in soldering applications. Atmospheric soldering allowed the recording of the melt dynamics with a camera placed over and transversal to the surface. The setup included a heating stage and an optical pyrometer for controlling the test temperature (Figure 12). 


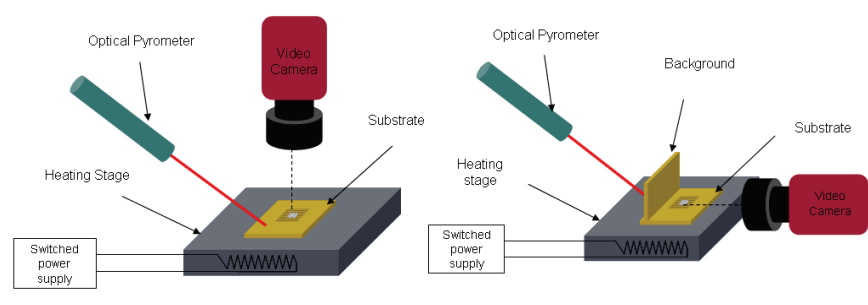

Figure 12: Soldering setup

In the case of the riblets texture, the drop moves along the preferential direction of the riblets and tends to occupy the entire area. Conversely, the mesh texture is capable to hold the drop with a stop-off like effect (Figure 13). The contact angle measurements are strongly influenced by the laser induced surface textured, as shown in the next section.
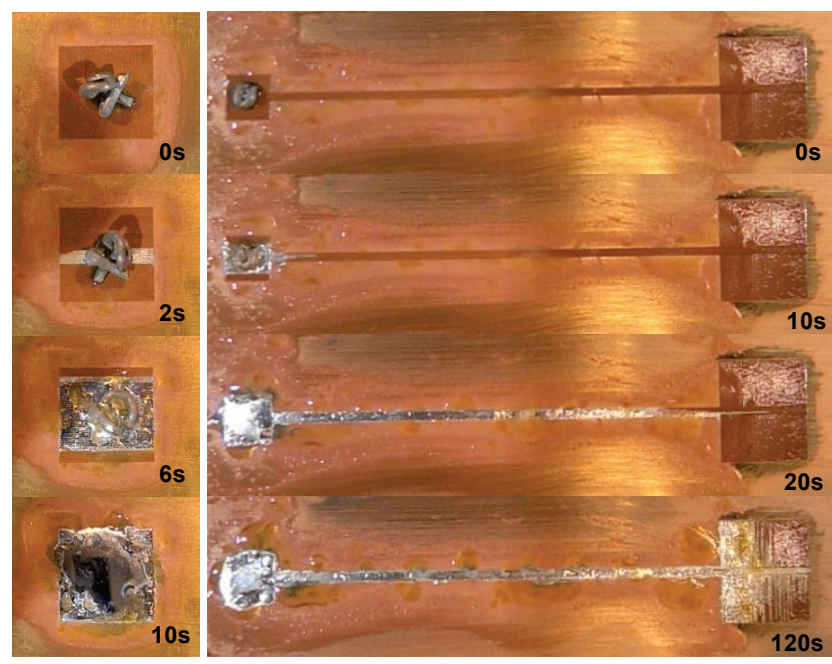

Figure 13: Sequences of images to show the spreading behaviour of solder on laser textured surfaces.

\section{Analysis of results}

Brazed joint samples with different surface conditions were prepared for testing their shear strength, to check how the surface preparation affects the mechanical quality of the bond. ISO 6892-1:2010 shear tensile test was used, as shown in the figure 14.
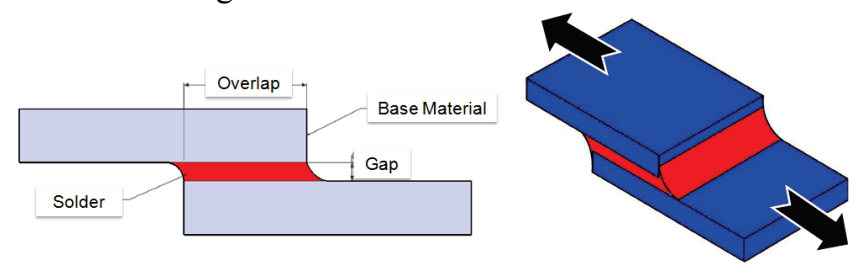

Figure 14: ISO 6892-1:2010 shear tensile test

Selected laser textured samples have been tested versus samples without surface texture. The results are shown in the following graph (Figure 15):

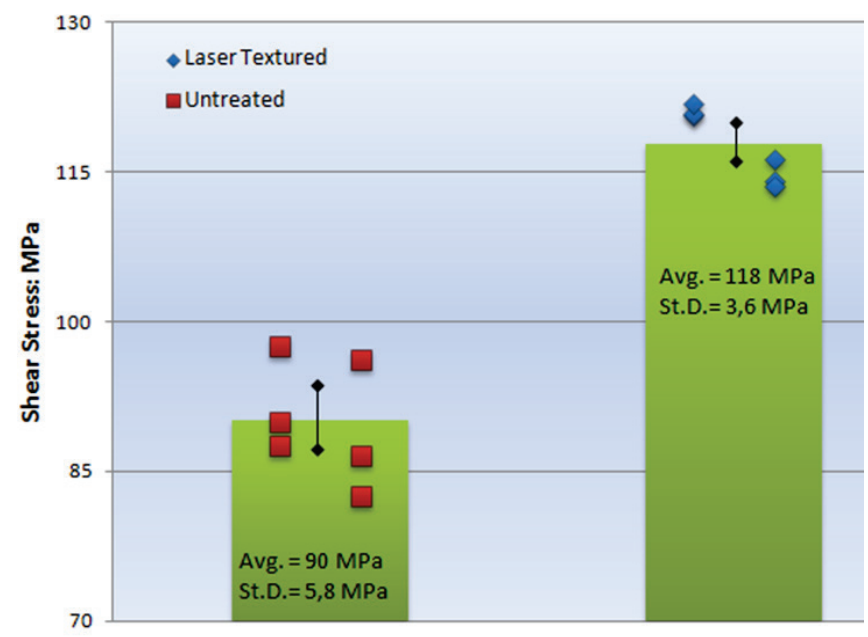

Figure 15: Shear tensile test results

Shear strength increases over $20 \%$ in laser textured samples, but it proves that the type of texture has low influence on the strength. It can be observed that the laser treatment reduces significantly the dispersion in the results, improving the dependability of the joint.

Wettability was also studied in soldering samples, in terms of reduction of the surface energy, improvement of the spreading, which can be measured as a low equivalent wetting contact. In the following images (Figure 16 and Figure 17) the textured samples results are shown versus unmodified surface.
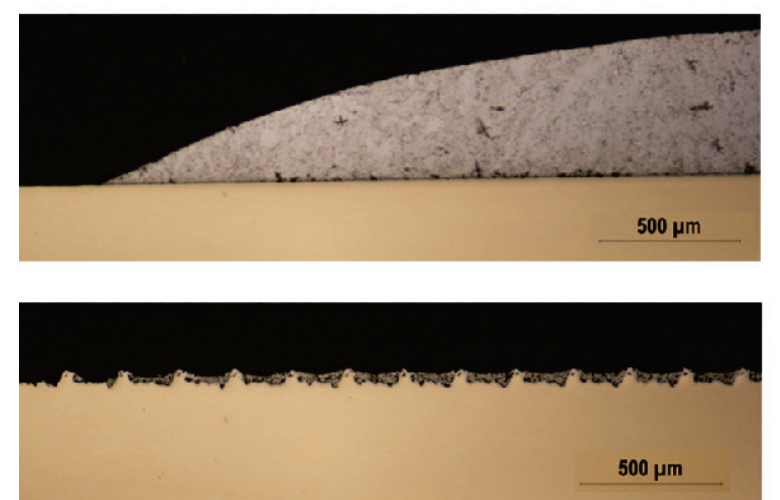

$\theta \sim 0^{\circ}$

Figure 16: Ribletted vs. unmodified surface

In Figure 16, molten Sn alloy moves in the direction of the riblets according to the desired behaviour. The result is an apparent contact angle nearly $0^{\circ}$, while the real contact angle is kept close to $20^{\circ}$. This allows large area spreading even under atmospheric conditions. 


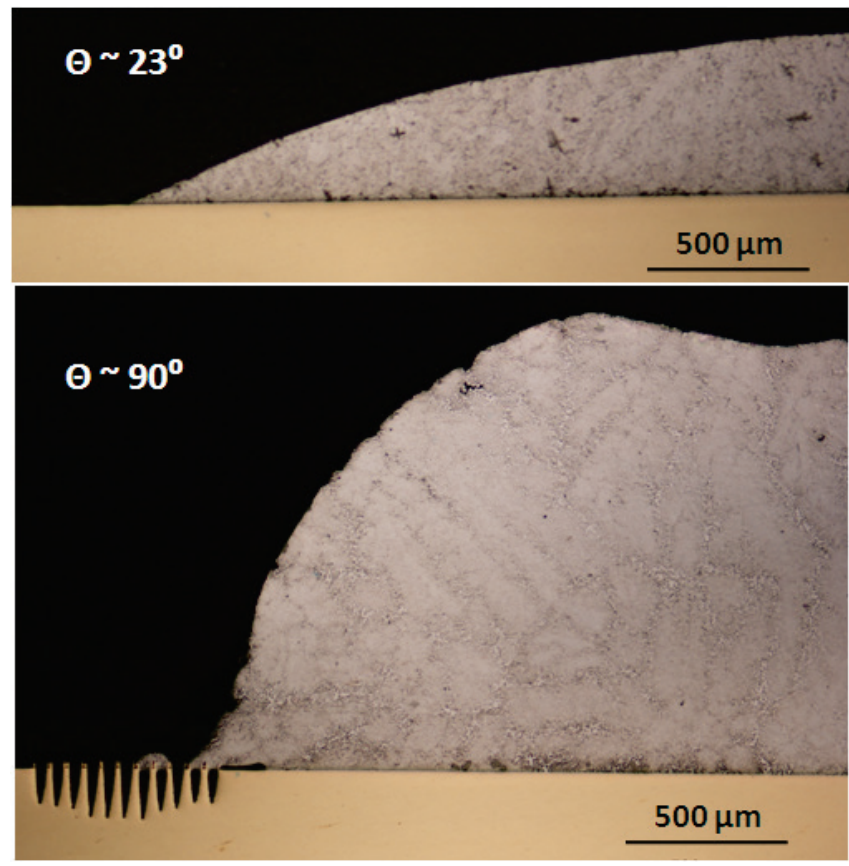

Figure 17: Contained (mesh) vs. unmodified surface

With a mesh texture of high aspect ratio, a strong Cassie-Baxter state is induced and the air-pocketing effectively retains the molten metal drop to create an apparent wetting angle close to $90^{\circ}$ (see Figure 17).

\section{Conclusions}

Custom adjustment of molten-metal wettability is feasible with laser texturing, and has been proven useful to conduct, improve or stop the flow of molten metal on a metallic surface.

The surface features needed for spread improvement or stopping were determined from a general theoretical framework, which resulted in geometrical requirement of high aspect ratio textures with small spatial frequency secondary roughness. These requirements were fulfilled with nanosecond and microsecond molten metal displacement texturing processes, depending on the target geometry, with ability to produce bimodal and anisotropic roughness distributions. This capability is relevant for brazing and soldering applications, to produce controlled surface conditions, improve the wetting of the desired area and avoid filler metal to overflow over undesired areas.

In the case of soldering, the surface texturing provided a way to effectively improve the wettability of a large area reducing the amount of solder material needed to produce the complete area wetting, and allowing a very precise placement of the wetting area. The results of this work have large potential for application in welding, soldering and coating applications.

\section{References}

[1] T. Handai, J. Riken, J. W. North, F. Minami and S. Seisan: J. Appl. Phys., 74, (1993) 1119.

[2] T. Handai, J. Riken, G. C. East, M. Nishi, J. W. North, F. Minami and S. Seisan: "Laser Precision Microfabrication" ed. by J. D. Center and D. Senri, (Publisher, Tokyo, 1999) p.864.

[3] Romero, P. et al., "High aspect ratio microfeatures with laser texturing in mixed ablative-melting regime", Micromachining and Microfabrication Process Technology XVII. (Ed. Maher, Resnick), SPIE, V 8248, pp. 82480C-18 (2012).

[4] Young, T., "An Essay on the Cohesion of Fluids", Phil. Trans., (1805), p. 65.

[5] Morita M, Koga T, Otsuka H and Takahara A. "Macroscopic-wetting anisotropy on the line-patterned surface of fluoroalkylsilane monolayers" Langmuir. 21 (2005) 911-8D.

[6] Quéré. "Wetting and roughness", Annual Review of Materials Research V38 (2008), p71-99

(Received: July 9, 2012, Accepted: February 28, 2013) 